\title{
Detailed balance analysis of solar thermophotovoltaic systems made up of single junction photovoltaic cells and broadband thermal emitters
}

\author{
A. Datas , C. Algora \\ Instituto de Energía Solar. Universidad Politécnica de Madrid, E.T.S.S. Telecomunicación, Ciudad Universitaria, 28040 Madrid, Spain - EU
}

\begin{abstract}
A B S T R A C T
This paper presents a detailed balance analysis of a solar thermophotovoltaic system comprising an optical concentrator, a cut-off broad band absorber and emitter, and single junction photovoltaic cells working at the radiative limit with an integrated back-side reflector in a configuration in which the cells enclose the emitter to form an optical cavity. The analysis includes the effect of multiple variables on the system performance (efficiency and electrical power density), such as the concentration factor, the emitter-to-absorber area ratio, the absorber and emitter cut-off energies, the semiconductor band-gap energy and the voltage of the cells. Furthermore, the effect of optical losses within the cavity such as those attributed to a back-side reflector with reflectivity lower than one or to a semi-open optical cavity is also included. One of our main conclusions is that for a planar system configuration (the emitter, the cells and the absorber have the same area) the combination of low concentration and a spectrally selective absorber provides the highest system efficiencies. The efficiency limit of this kind of systems is $45.3 \%$, which exceeds the Shockley-Queisser limit of $40.8 \%$ (obtained for a single junction solar cell, directly illuminated by the sun, working under maximum concentration and with an optimized band-gap). This system also has the great benefit of requiring a very low concentration factor of 4.4 suns.
\end{abstract}

\section{Introduction}

Solar thermophotovoltaic (STPV) systems use solar radiation to heat an intermediate material. This material then radiates toward the photovoltaic (PV) cells where the radiation is converted into electricity. The surface of the intermediate material that absorbs the sunlight is the absorber and the surface that radiates toward the cells is the emitter. The emitter and the PV cells make up the thermophotovoltaic (TPV) optical cavity where the radiation is confined (Fig. 1).

This configuration provides one of the highest limiting efficiencies among all of the proposed photovoltaic approaches $(85.4 \%)[1,2]$; the efficiency equals that of an ideal solar-thermal engine and is slightly below the efficiency limit of a tandem cell with an infinite number of band-gaps (86.8\%) [3]. However, the efficiency limit of a STPV system is obtained under a set of very restrictive constraints. First, it is assumed that the absorber only exchanges photons with the sun and with no other part of the sky (which implies the use of an intermediate optical system with the maximum concentration factor [4] or a special kind of angular selective absorber [5,6]). Second, the emitter-to-cells photon exchange must be strictly monochromatic (with photon energies equaling the band-gap energy of the PV semiconductor). Finally, the emitter area must be much larger than that of the absorber (approaching infinity) to compensate for the very narrow spectral range of emission (approaching zero); thus, the extracted electrical power density is very low (approaching zero) [7].

These constraints introduce significant technological issues that make this efficiency unachievable in practice. First, very high concentration factors imply very low tolerances in both the sun tracking system and in the optical components that make up the concentrator. At present, achievable tolerances limit the practically achievable geometrical concentration factor to approximately $\sim 1000 \times[8]$. Second, despite the fact that a number of proposed designs for quasi-monochromatic emitters have been developed, many of the issues concerning their performance at high temperatures have still not been resolved [9]. Finally, very large emitter-to-absorber area ratios require the use of $3 D$-geometry emitters with a very large aspect ratio $[10,11]$, which introduces problems with the TPV optical cavity design and the integration of PV cell arrays.

In this paper we analyze STPV systems consisting of broadband cut-off thermal emitters and single junction PV cells. Under specific conditions that are described in this the paper, these kinds of systems allow both high efficiency and high electrical power density in configurations with a low emitter-to-absorber 


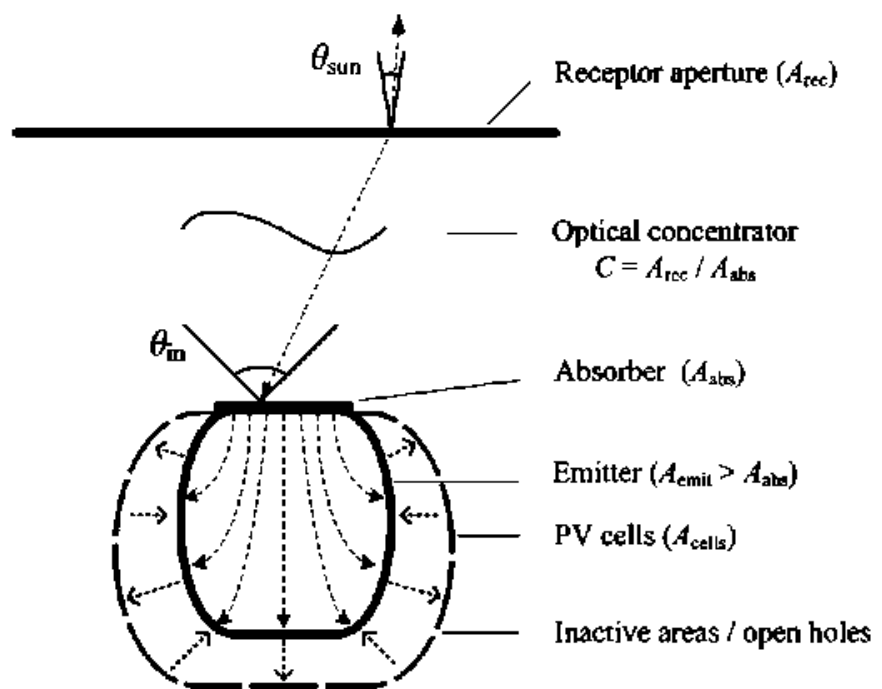

Fig 1. The radiation exclange at the absorberfemitter element of an STPV system.

area ratio and concentration factor. In addition, they can be designed to have low sensitivity to optical losses within the TPV optical cavity. Therefore, in this paper we present practical alternatives to develop high efficiency STPV systems. The analysis is based on the detailed balance theory $[12,13]$, which allows us to calculate the upper limits of efficiency.

\section{Theory}

\subsection{Spatial/spectral extension of radiatian}

The photon flux $(\dot{N})$ and the energy flux (or power density, $\dot{E}$ ) of photons with electrochemical potential $\mu$ and energy between $\varepsilon_{1}$ and $\varepsilon_{2}$ that are emitted by a surface at temperature $T$ in a vacuum in the normal direction and per unit of solid angle are given by the generalized Planck equation $[1,14]$ :

$\dot{N}\left(\varepsilon_{1}, \varepsilon_{2}, T, \mu\right) \equiv \int_{\varepsilon_{1}}^{\varepsilon_{2}} \mathrm{e}(\varepsilon) \cdot \dot{n}\left(\varepsilon_{+} T, \mu\right) d \varepsilon \equiv \frac{2}{h^{3} c^{2}} \int_{\varepsilon_{1}}^{\varepsilon_{2}} \mathrm{e}(\varepsilon) \cdot \frac{\varepsilon^{2} d \varepsilon}{\exp \left(\frac{\varepsilon-\mu t}{k T}\right)-1}$

$\grave{E}\left(\varepsilon_{1}, \varepsilon_{2}, T, \mu\right) \equiv \int_{\varepsilon_{1}}^{\varepsilon_{2}} e(\varepsilon) \cdot \dot{e}(\varepsilon, T, \mu) d \varepsilon \equiv \frac{2}{h^{3} c^{2}} \int_{\varepsilon_{1}}^{\varepsilon_{2}} e(\varepsilon) \cdot \frac{\varepsilon^{3} d \varepsilon}{\exp \left(\frac{\varepsilon-\mu}{k T}\right)-1}$

where $h$ is Planck's constant, $k$ is Boltzmann's constant, $c$ is the speed of light in a vacuum, $e(\varepsilon)$ is the spectral emissivity of the sutface and $\dot{n}(\varepsilon, T, \mu)$ and $\dot{e}(\varepsilon, T, \mu)$ denote the spectral photon and energy fluxes in the normal direction and per unit of solid angle, respectively. In this paper it is assumed that $e(\varepsilon)=1$ for the interval $\left(\varepsilon_{1}-\varepsilon_{2}\right)$. The total photon and energy flux emitted by a surface $d A$ immersed in a medium of refraction index $n_{\text {out }}$ in a solid angle $d \Omega$ and in a direction defined by an angle $\theta$ with the surface normal (Fig. 2) is given by $\dot{N}\left(\varepsilon_{1}, \varepsilon_{2}, T, \mu\right) d H$ and $\dot{E}\left(\varepsilon_{1}, \varepsilon_{2}, T, \mu\right) d H$, respectively, where $d H$ is the differential of the Lagrange invariant or étendue (extension) [4] given by: $\mathrm{d} H \equiv n_{\text {out }}^{2} \cos \theta d A d \Omega=\mathrm{u}_{\text {out }}^{2} \cos \theta \sin \theta d A d \theta d \varphi$. Integration over $A,\left(\theta_{\min }, \theta_{\max }\right)$ and $\left(\varphi_{\min }, \varphi_{\max }\right)$ produces

$H\left(\mathrm{uj}_{\mathrm{out}}, A, \theta_{\min }, \theta_{\max }, \varphi_{\min }, \varphi_{\max }\right)$

$$
\begin{aligned}
& =\mathrm{n}_{\text {out }}^{2} \cdot \int_{A} \int_{\theta_{\mathrm{Imin}}}^{\theta_{\max }} \int_{\varphi_{\text {mix }}}^{\varphi_{\text {max }}} \cos \theta \sin \theta d A d \theta d \varphi \\
& =\frac{\mathrm{n}_{\text {out }}^{2}}{2} \cdot A \cdot\left(\varphi_{\max }-\varphi_{\text {min }}\right) \cdot\left(\sin ^{2} \theta_{\max }-\sin ^{2} \theta_{\text {min }}\right)
\end{aligned}
$$

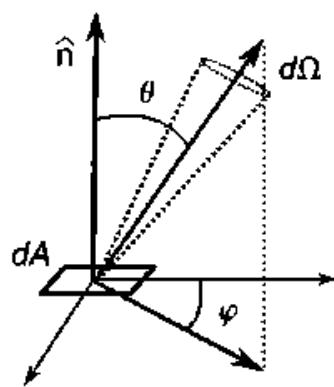

Fig. 2. The differential solid angle $d \Omega$ of emission from a surface $d A$ defining an angle $\theta$ with the surface normal vector $\hat{n}$

In geometrical optics, $H$ represents the volume that a beam fills in the phase-space domain [4]: thus, it represents the spatial/angular extension of emission, and it must be conserved in a non-lossy optical system. Eqs. (1)-(3) are used to determine the radiative exchange between all the components that constitute the STPV system.

\subsection{Sunlight receptor}

In this paper, the radiation leaving the sun's sulface is approximated by the emission of a black-body at $6000 \mathrm{~K}$, which provides the spectral distribution of the photons described in Eqs. (1) and (2). The radiation intercepted by the Earth's surface is restricted to a vely narrow solid angle that depends on the SunEarth distance (approximately $150 \times 10^{6} \mathrm{~km}$ ) and the sun's diameter (approximately $1.4 \times 10^{6} \mathrm{~km}$ ). It represents an angle of $\theta_{\text {sun }}=0.267^{\circ}$. Then, because the refractive index of air is close to one and assuming that no-optical losses and/or dispersion occurs in the atmosphere, the étendue $H$ of the light coming from the sun that intercepts a receptor aperture of area $A_{\text {rec }}$ (given by Eq. (3)) is $H_{\text {sun }}=A_{\text {rec }} \cdot \pi \sin ^{2}\left(\theta_{\text {sun }}\right)$; therefore, the sunlight energy flux at the Earth's surface is approximately $0.16 \mathrm{~W} / \mathrm{cm}^{2}$.

To take advantage of the sunlight, an intermediate sunlight receptor must be used to transfer this energy to the photovoltaic cells to produce useful work. The simplest receptor consists of a material (the absorber) that is able to absorb the radiation in the spatial/spectral extension of the incoming sunlight. However, every material able to absorb can also emit, as stated by Kirchhoff's law of radiation, and this emission represents losses. These losses depend on the spatial-spectral emission intrinsic properties of the material and its temperature. Therefore, to minimize these losses, either the spatial (area and angle) and/or the spectral extension of the emission must be restricted without reducing the absorption.

\subsubsection{Spatial restriction (aptical concentration)}

Despite the fact that some materials can behave as angleselective absorbers/emitters [5], in most cases they behave as diffuse (Lambertian or isotropic) emitters, which means that the intensity (power per unit of solid angle) of the radiation leaving their surfaces is independent of direction. Therefore, in these materials, the only way to reduce emission losses is to reduce the absorber area, which requires the use of an optical concentration system between the receptor and the absorber in order to maintain

\footnotetext{
${ }^{\prime}$ According to the more realistic sun spectra shown in the ASTM terrestrial reference standards, the sunlight energy flux at the Earth is closer to $0.13 \mathrm{~W} / \mathrm{cm}^{2}$ (AMO). Nevertheless, in order to allow the results to be compared with other studies, we abide by the convention of using the approximated spectrum of a black body at $6000 \mathrm{~K}$, which reaches the Earth surface within an angle of $0.267^{\prime \prime}$.
} 
the same input power. The ratio between the receptor and the absorber area is termed the concentration factor $C=A_{\text {red }} / A_{\text {abs }}$, and it can be formulated as a function of the edge angles of the beams at the receptor and the absorber planes (Fig. 1): $C=A_{\text {rec }} / A_{\text {abs }}=\sin ^{2}\left(\theta_{\text {in }}\right) / \sin ^{2}\left(\theta_{\text {sun }}\right)$; therefore, $C$ has an upper limit when $\theta_{\text {in }}=\pi / 2$ of $C_{\max }=1 / \sin ^{2}\left(\theta_{\text {sun }}\right)=46,050$ (assuming a material with $n_{\text {out }}=1$ surrounding the absorber). In this situation, all the radiation originating from the absorber leaves the receptor aperture within an angle $\theta_{\text {sun }}$; thus, the absorber exchanges photons with the sun but no other portion of the sky.

\subsubsection{Spectral restriction (Spectral selective absorber)}

Because the absorber temperature is lower than that of the sun (and therefore the absorber emission spectrum is shifted to lower photon energies), it is reasonable to restrict the absorption/ emission of the absorber to photons with energies higher than a specific threshold, $\varepsilon_{\mathrm{ca}}[15]$. Because the sun and absorber spectra overlap, the choice of $\varepsilon_{\mathrm{ca}}$ must be determined by the trade-off between the absorbed and emitted energy, which strongly depends on the absorber temperature. These kinds of spectralselective absorbers usually consist of nanostructured surfaces with feature sizes on the order of $100 \mathrm{~nm}$ to $1 \mu \mathrm{m}$ depending on the desired cut-off energy $[16,17]$.

\subsection{PV cells}

The PV cells described in this paper consist of a diode (pn junction) made of a single semiconductor with an energy band gap $\varepsilon_{\text {gap }}$ (Fig. 3) the front side of which is completely uncovered to allow the light to penetrate into the semiconductor. In addition, the following assumptions are held:

- The cells work at the radiative limit; in other words, only radiative recombination takes place and each photon can generate only one electron-hole pair (and vice versa) [12,13].

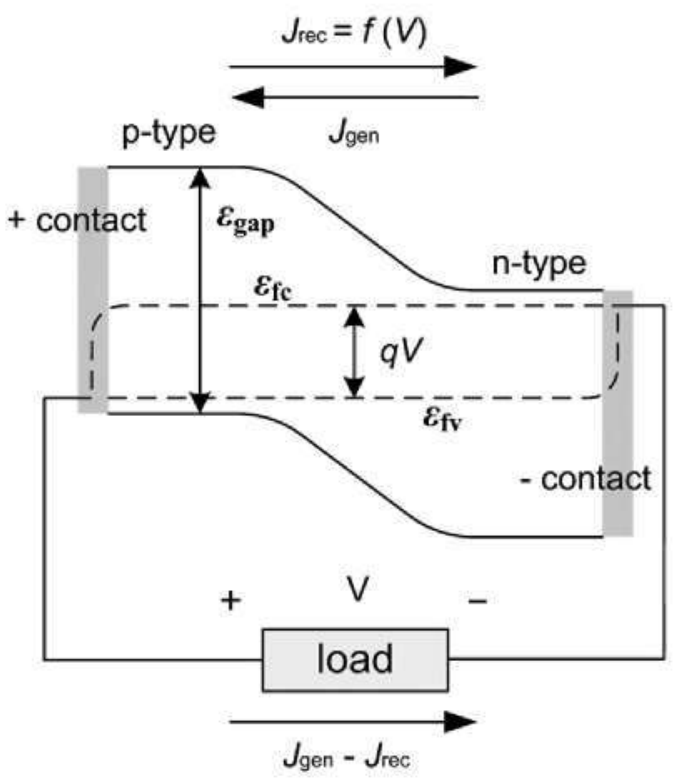

Fig. 3. The bands diagram of an ideal illuminated PV cell for which the quasiFermi level splitting $\varepsilon_{\mathrm{fc}}-\varepsilon_{\mathrm{fv}}$ is constant and equals $q V$ everywhere in the cell. The current delivered to an external load comes from the balance between generation and recombination of carriers, where the generation process is related to the absorption/collection of the incident photons and the recombination process represents the luminescent radiation (photons with chemical potential $\mu=q V$ ) leaving the cells.
- The free electrons and holes have infinite mobility within the semiconductor (zero resistance); thus, quasi-Fermi level splitting can be assumed to be constant everywhere in the cell and equal to $q V[13]$ (where $V$ is the external cell voltage).

- The cells are covered with an ideal anti-reflective coating (ARC); thus, all the photons impinging on the cell surface penetrate into the semiconductor.

- The cells have a specular back-side reflector (BSR) of reflectivity $\rho_{\text {bsr }}$ (Figs. 4 and 5).

- The photon-to-electron conversion takes place in a region of the semiconductor, referred to as the diode active layers (Figs. 4 and 5), where all the photons with energies above $\varepsilon_{\text {gap }}$ are absorbed (i.e. the diode spectral emissivity/absorptivity $\alpha_{d}(\varepsilon)$ equals zero if $\varepsilon<\varepsilon_{\text {gap }}$ and equals one otherwise).

According to the first two assumptions, both the photon and energy fluxes per unit of solid angle emitted due to radiative recombination between electrons and holes within the cells can be formulated by Eqs. (1) and (2) making $\mu=q V, \varepsilon_{1}=\varepsilon_{\text {gap }}$ and $\varepsilon_{2} \rightarrow \infty$. Under these conditions, no electrons are lost due to any mechanism other than radiative recombination. Therefore, the current delivered to an external load (Fig. 3) can be calculated as the balance of the photon fluxes within the cells; that is, the flux of delivered electrons (electrical current) must equal the balance between the absorbed photon flux (generation) and the emitted photon flux (radiative recombination). From this balance, the delivered electric current is formulated as a function of the voltage (i.e., $J=J(V)$ ), and the maximum electric power delivered to an external load (i.e., the maximum power point (MPP)) of the $J-V$ curve is found for a pair $\left(V_{\text {mpp }} J_{\text {mpp }}\right)$ that maximizes $P=V \cdot J$.

Because the photon flux impinging on the cells depends on the external conditions (i.e., the concentration factor, the TPV optical cavity configuration and the absorber/emitter characteristics), the analytical expression for $J=J(V)$ must be calculated by considering all the characteristics of the complete system, as shown in Sections 2.4 and 2.6 .

\subsection{TPV optical cavity}

In a TPV system, the PV cells enclose the emitter to form an optical cavity (Fig. 1), which consists of the emitter, the cells and the inactive areas or open holes. In this paper we assume that the emission from the emitter is restricted to photons with energies above $\varepsilon_{\mathrm{ce}}$ (which is the emitter cut-off energy). In other words, the emitter spectral emissivity/absorptivity $\alpha_{\mathrm{e}}(\varepsilon)$ equals zero if $\varepsilon<\varepsilon_{\mathrm{ce}}$ and equals one otherwise. To determine the radiative exchange within this cavity, the net-radiation method $[18,19]$ can be used. In this method, a set of $2 N$ equations (where $N$ is the number of surfaces within the cavity) is established. Each of these equations results from the fact that the energy flux (energy per unit of time and area) leaving a surface is composed of directly emitted as well as reflected and transmitted energy. In our case, the TPV cavity (Fig. 4) can be modeled using four surfaces $(N=4)$ : the emitter, the outside boundary of the cells (or ARC), the + side of the diode active layers (the one facing the TPV cavity) and the - side of the diode active layers (the one facing the BSR). For this cavity, we assume that:

- Every surface (except the BSR) emits and reflects in a diffuse manner.

- All of the surfaces are isothermal.

- The emission originating from surfaces at room temperature and with $\mu=0$ (BSR and inactive areas within the cavity) is negligible.

- The emitter is convex (it cannot see itself) and opaque. 

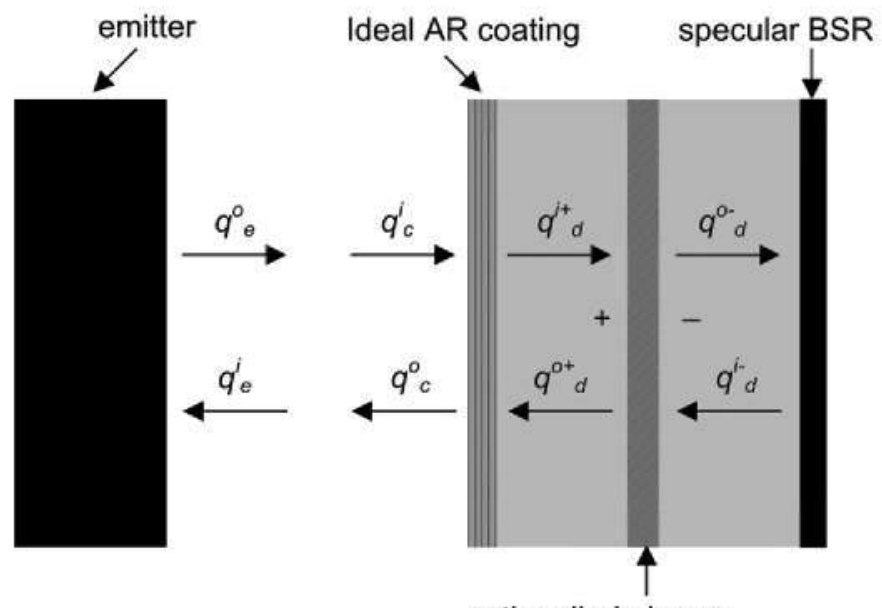

active diode layers

Fig. 4. Energy fluxes within a TPV optical cavity consisting of a black-body emitter and BSR PV cells.

The first assumption allows us to simplify the cavity analysis because the fraction of the energy flux leaving a surface $x$ that impinges a surface $y$ when surface $z$ is a shadowing obstacle can be expressed by a purely geometrical factor, the view factor $F_{x y}^{(z)}[18]$.

When the emissivity of the surfaces involved is spectrally dependent (as it is in our case), this method must be evaluated for a single photon energy, and therefore, spectral energy fluxes must be considered. Afterwards, these spectral energy fluxes must be integrated over the range of energies of interest to provide the total energy flux. To evaluate this method it is convenient to determine the radiation exchange within the PV cells and the TPV cavity.

\subsubsection{Radiation within PV cells}

Fig. 5 shows a detailed photon exchange within the PV cells, where two sources of photons exist: those that come from an external source $(A)$, with an étendue $H_{\mathrm{ext}}=\pi A_{\text {cells }}$ (assumed diffuse) and those generated inside the cells due to radiative recombination (G) and (I), with an étendue $H_{\text {int }}=\pi n_{\text {int }}^{2} A_{\text {cells }}$ (also assumed diffuse) where $n_{\text {int }} \sim 3.5$ for most of the semiconductors. Each kind of photon follow different paths within the cell:

The spectral energy flux of photons impinging on the cells (A), noted by $q_{\varepsilon, c}^{i}(\varepsilon)$, is transferred inside the cell (B). Because of the ideal ARC, no photons are lost from $(A)$ to $(B)$. Nevertheless, to maintain a constant étendue $\left(H_{\mathrm{ext}}\right)$, the angular extension of light is reduced from $\pi / 2$ (isotropic) to $\theta_{\max }=\arcsin \left(1 / n_{\text {int }}\right)$ because of the change in the refractive index from 1 to $n_{\text {int }}$. Therefore, all these photons contribute to the spectral energy flux at the + side of the diode active layers $q_{\varepsilon, d}^{i+}(\varepsilon)$ (last term of Eq. (8)). Of these photons, those that have $\varepsilon>\varepsilon_{\text {gap }}$ are absorbed in the diode active layers and generates an electron-hole pair. The rest of the photons (with $\varepsilon<\varepsilon_{\text {gap }}$ ) cross the diode active layers without being absorbed, which contributes to the outgoing spectral energy flux at the - side of the diode active layers $q_{\varepsilon, d}^{o-}(\varepsilon)$ (the last term of Eq. (5)). These photons then reach the BSR (C), and a portion $\rho_{\mathrm{bsr}}$ is reflected back (D) keeping the same angular extension (because the reflector is assumed to be specular). These photons contribute to the spectral energy flux that impinges on the - side of the diode active layers $q_{2, d}^{i-}(\varepsilon)$ (the last term of Eq. (9)). Because all these photons have $\varepsilon<\varepsilon_{\text {gap }}$, they cross the diode active layers

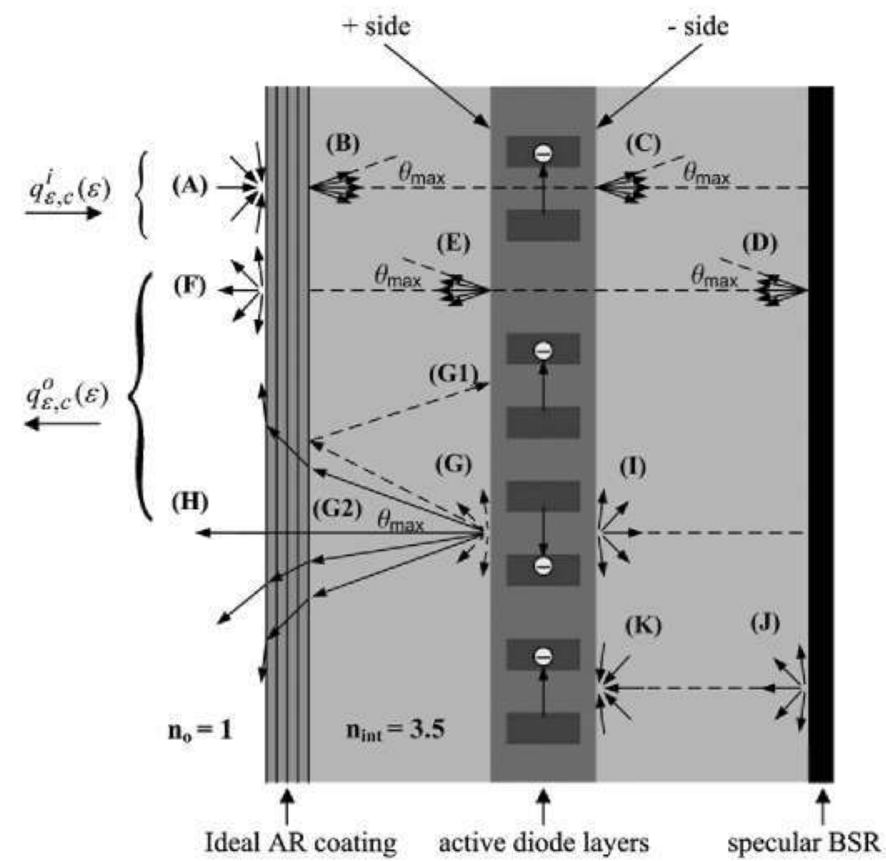

Fig. 5. Radiative exchange within the ideal BSR PV cell.

without being absorbed (E), and contribute to the spectral energy flux leaving the + side of the diode active layers $q_{\varepsilon, d}^{o+}(\varepsilon)$ (the last term of Eq. (4)). Finally they leave the cell, again as isotropic radiation ( $F$ ) and contribute to the spectral energy flux leaving the cell surface $q_{\varepsilon, c}^{o}(\varepsilon)$ (the last term of Eq. (6)).

All of the photons originating at the diode active layers have $\varepsilon>\varepsilon_{\text {gap }}, \mu=q V$, and they are emitted from both $+(\mathrm{G})$ and $-(\mathrm{I})$ sides of the diode active layers. Of the photons emitted from the + side (G) (the first term of Eq. (4)), only those within an angle $\theta<\theta_{\max }(\mathrm{G} 2)$ come out of the cell as isotropic radiation (H), and contribute to the outgoing spectral energy flux at the cells $q_{\delta, c}^{o}(\varepsilon)$ (the first term of Eq. $(6)$ ). The rest of the photons (G1) impinge on the + side of the diode active layers (the first term of Eq. (8)) and are reabsorbed. Of the photons emitted from the - side (I) (the first term of Eq. (5)), a portion $\rho_{\text {bsr }}$ impinge on the + side of the diode active layers (the first term of Eq. (9)) and are reabsorbed, and a portion $1-\rho_{\text {bsr }}$ is lost in the BSR.

\subsubsection{Radiation within the TPV cavity}

The spectral energy flux of the photons that impinges on the cells $q_{\varepsilon, c}^{i}(\varepsilon)$ (Eq. (10)) has two components: the photons coming from the emitter $q_{\varepsilon, e}^{o}(\varepsilon)$ and the photons coming from the cells themselves $q_{\varepsilon, c}^{o}(\varepsilon)$. The ratio of each contribution is given by the emitter-to-cells view factor $F_{\text {ec }}$ and the cells-to-cells view factor when the emitter is a shadowing obstacle $F_{c c}^{(e)}$ (the first and second terms of Eq. (10), respectively). Similarly, the spectral energy flux impinging on the emitter $q_{\varepsilon_{e}}^{i}(\varepsilon)$ is simply given by the fraction of the spectral energy flux of photons leaving the cells that intercepts the emitter (Eq. (11)). Finally, the outgoing spectral energy flux leaving the emitter surface $q_{\varepsilon, e}^{o}(\varepsilon)$ has two components: the photons originating directly from the emitter surface (the first term of Eq. (7)) and the photons reflected at the emitter (the second term of Eq. (7)).

$$
q_{\varepsilon, d}^{o+}(\varepsilon)=\pi n_{\mathrm{inr}}^{2} \alpha_{\mathrm{d}}(\varepsilon) \cdot \dot{e}\left(\varepsilon, T_{\text {cells }}, q V\right)+\rho_{\mathrm{bsr}}\left(1-\alpha_{\mathrm{d}}(\varepsilon)\right) \cdot q_{\varepsilon, c}^{i}(\varepsilon)
$$




$$
\begin{aligned}
& q_{\varepsilon, \dot{d}}^{\boldsymbol{o}}(\varepsilon)=\pi \mathrm{u}_{\mathrm{int}}^{2} \alpha_{\mathrm{d}}(\varepsilon) \cdot \dot{e}\left(\varepsilon, T_{\text {cells }}, q^{V}\right)+\left(1-\alpha_{\mathrm{d}}(\varepsilon)\right) \cdot q_{\varepsilon, c}^{\mathrm{j}}(\varepsilon) \\
& q_{\varepsilon, c}^{o}(\varepsilon)=\pi \alpha_{\mathrm{d}}(\varepsilon) \cdot \dot{e}\left(\varepsilon, T_{\mathrm{ce}] l s}, q V\right)+\rho_{\mathrm{bsr}}\left(1-\alpha_{\mathrm{d}}(\varepsilon)\right) \cdot q_{\varepsilon, c}^{i}(\varepsilon) \\
& q_{\varepsilon, e}^{o}(\varepsilon)=\pi \alpha_{\mathrm{e}}(\varepsilon) \cdot \dot{e}\left(\varepsilon, T_{\mathrm{emit}}, 0\right)+\left(1-\alpha_{\mathrm{e}}(\varepsilon)\right) \cdot q_{\varepsilon, e}^{i}(\varepsilon) \\
& q_{\varepsilon, d}^{i+}(\varepsilon)=\pi\left(n_{\mathrm{int}}^{2}-1\right) \alpha_{d}(\varepsilon) \cdot \dot{e}\left(\varepsilon, T_{\text {cells }}, q V\right)+q_{\varepsilon, c}^{i}(\varepsilon) \\
& q_{\varepsilon, d}^{i-}(\varepsilon)=\pi \mathrm{u}_{\mathrm{jnt}}^{2} \alpha_{\mathrm{d}}(\varepsilon) \rho_{\mathrm{bss}} \cdot \dot{e}\left(\varepsilon, T_{\mathrm{cell}}, q^{V}\right)+\rho_{\mathrm{bsr}}\left(1-\alpha_{\mathrm{d}}(\varepsilon)\right) \cdot q_{\varepsilon, c}^{i}(\varepsilon) \\
& q_{\varepsilon, c}^{i}(\varepsilon)=F_{\mathrm{ce}} \cdot q_{\varepsilon, e}^{o}(\varepsilon)+F_{c \mathrm{c}}^{\mathrm{e})} \cdot q_{\varepsilon, c}^{o}(\varepsilon) \\
& q_{\varepsilon, e}^{i}(\varepsilon)=F_{\mathrm{ec}} \cdot q_{\varepsilon, c}^{o}(\varepsilon)
\end{aligned}
$$

Introducing Eqs. (10) and (11) into Eqs. (6) and (7) and integrating over all the possible energies of photons $(0<\varepsilon<\infty)$ produces

$$
\begin{aligned}
q_{e}^{i}-q_{e}^{o}= & \int_{0}^{\infty}\left[q_{\varepsilon, e}^{i}(\varepsilon)-q_{\varepsilon, e}^{o}(\varepsilon)\right] \mathrm{d} \varepsilon=\pi \cdot\left[F_{\mathrm{ec}} \cdot \dot{E}\left(\varepsilon_{\mathrm{gap}}, \infty, T_{\mathrm{ce}] \mathrm{s}}, q V\right)\right. \\
& \left.+\frac{A_{\mathrm{emit}}}{A_{\mathrm{ce} l \mathrm{ls}}} \cdot \frac{\rho_{\mathrm{bsr}} F_{\mathrm{ec}}^{2}}{1-\rho_{\mathrm{bsr}} F_{\mathrm{cc}}^{\mathrm{e} !}} \cdot \dot{E}\left(\varepsilon_{\mathrm{ce}}, \varepsilon_{\mathrm{gap}}, T_{\mathrm{emit}}, 0\right)-\dot{E}\left(\varepsilon_{\mathrm{ce}}, \infty, T_{\mathrm{emir}}, 0\right)\right]
\end{aligned}
$$

which represent the energy balance at the emitter surface, which is usually negative (output energy surpasses the input energy) because the emitter usually transfers energy to the cells and not vice versa. The first two terms of Eq. (12) account for the incoming energy flux: the first one accounts for the photons emitted by the diode active layers (with $\mu=q V$ ) and the second one for the sub band-gap photons $\varepsilon_{\mathrm{ce}}<\varepsilon<\varepsilon_{\text {gap }}$ returned to the emitter after reflection by the BSR. The third term represents the total energy flux leaving the emitter.

\subsection{The energy balonce equation}

In a STPV system, the net absorbed energy from the sun (Section 2.2) is transferred to the emitter and then to the PV cells that convert the radiation into electricity. The system efficiency greatly depends on the absorber/emitter temperature, which comes from the solution of the energy balance equation at the absorber/emitter component. Assuming that the absorber and the emitter have the same temperature $T_{a b s}=T_{\text {emit }}$ (which assumes infinite thermal conductivity between them), we can write

$$
\begin{aligned}
& H\left(1, A_{\mathrm{abs}}, 0, \theta_{\mathrm{in}}, 0,2 \pi\right) \cdot\left[\dot{E}\left(\varepsilon_{\mathrm{ca}}, \infty, T_{\mathrm{sun}}, 0\right)-\dot{E}\left(\varepsilon_{\mathrm{ca}}, \infty, T_{\text {emit }}, 0\right)\right] \\
& \quad+H\left(1, A_{\mathrm{abs}}, \theta_{\mathrm{in},}, \pi / 2,0,2 \pi\right) \cdot\left[\dot{E}\left(\varepsilon_{\mathrm{ca}}, \infty, T_{s k y}, 0\right)-\dot{E}\left(\varepsilon_{\mathrm{ca}}, \infty, T_{\text {emit }}, 0\right)\right] \\
& \quad+A_{\mathrm{emit}} \cdot\left[q_{\mathrm{e}}^{\mathrm{i}}-q_{\mathrm{e}}^{0}\right]=0
\end{aligned}
$$

In this equation, the first term accounts for the radiation exchange between the sun and the absorber (limited to photons with $\varepsilon>\varepsilon_{c a}$ and within a cone forming an angle $\theta_{\text {in }}$ relative to the surface normal, Fig 1 ). The second term represents the radjation exchange between the absorber and the sky, which is usually a loss when $T_{\text {sky }}<T_{\text {emit- }}$ The third term represents the eneigy balance at the emitter surface (Eq. (12)), which depends on the optical cavity that encloses the emitter and is discussed in Section 2.4. The solution of Eq. (13) provides the equilibrium emitter temperature $\left(T_{\text {ernit }}\right)$ when $T_{\text {sun }}=6000 \mathrm{~K}$ and $T_{\text {sly }}=T_{\text {env }}=T_{\text {cells }}=300 \mathrm{~K}$.

After some algebraic operations and introducing Eq. (12) into Eq. (13) we obtain the final energy balance equacian for the absorber/emitter component that includes the effects of the concentrator/absorber (Section 2.2), the PV cells (Section 2.3) and the TPV cavity (Section 2.4):

$$
\begin{aligned}
& \frac{C}{C_{\max }} \cdot\left[\dot{E}\left(\varepsilon_{\mathrm{ca}}, \infty, T_{\text {sun }}, 0\right)-\dot{E}\left(\varepsilon_{\mathrm{ca}}, \infty, T_{\text {emit }}, 0\right)\right] \\
& +\left(1-\frac{C}{C_{\max }}\right) \cdot\left[\dot{E}\left(\varepsilon_{\mathrm{ca},}, \infty, T_{\text {sky }}, 0\right)-\dot{E}\left(\varepsilon_{\mathrm{ca}}, \infty, T_{\mathrm{emit}}, 0\right)\right] \\
& +\left(\frac{A_{\text {emir }}}{A_{\text {abs }}}\right) \cdot\left[F_{\mathrm{ec}} \cdot \dot{E}\left(\varepsilon_{\mathrm{gap}}, \infty, T_{\text {cells }}, q V\right)-\dot{E}\left(\varepsilon_{\mathrm{ce}}, \infty, T_{\text {emit }}, 0\right)\right. \\
& \left.+\frac{A_{\text {emit }}}{A_{\text {cells }}} \cdot \frac{\rho_{\text {bss }} F_{\text {es }}^{2}}{1-\rho_{\text {bss }} F_{c c}^{(e)}} \cdot \dot{E}\left(\varepsilon_{c e}, \varepsilon_{\text {gap }}, T_{\text {emit }}, 0\right)\right]=0
\end{aligned}
$$

\subsection{Photocurrent density ond efficiency}

Once the energy balance equation (14) is solved, the photocurrent density can be calculated as the net rate of photon absorption at the diode active layers (see Section 2.3), which results in the following equation:

$$
\begin{aligned}
J= & \frac{q}{A_{\text {cells }}} \cdot[\text { incoming photon flux-outgoing photon flux }] \\
& =q \cdot \int_{\varepsilon_{\text {xap }}}^{\infty} \frac{1}{\varepsilon} \cdot\left[q_{\varepsilon, d}^{i+}(\varepsilon)+q_{\varepsilon, d}^{i-}(\varepsilon)-q_{\varepsilon, d}^{o+}(\varepsilon)-q_{\varepsilon, d}^{o-d}(\varepsilon)\right] d \varepsilon
\end{aligned}
$$

Substituting the solution of Eqs. (4)-(11) into Eq. (15) produces

$$
\begin{aligned}
J= & J(V)=q \pi \cdot\left[\left(A_{\text {emit }} / A_{\text {cells }}\right) \cdot F_{\text {ec }} \cdot \dot{N}\left(\varepsilon_{\text {gap }}, \infty, T_{\text {emit }}, 0\right)\right. \\
& -\left(1-F_{c c}^{(e)}\right) \cdot \dot{N}\left(\varepsilon_{\text {gap }}, \infty, T_{c e l l s}, q V\right) \\
& \left.-11_{\text {int }}^{2}\left(1-\rho_{\text {bss }}\right) \cdot \dot{N}\left(\varepsilon_{\text {gap }}, \infty, T_{c e l l s}, q V\right)\right]
\end{aligned}
$$

where the first term accounts for the generation of electron-hole pairs and the last two terms account for the radiative recombination from the + and - sides of the diode active layers, respectively. Then, the STPV system efficiency is calculated by dividing the electrical power extracted from the PV cells $\left(A_{\text {cells }} \cdot J \cdot V\right)$ by the incoming sun radiation, to obtain

$\eta=\frac{A_{\text {cells }} \cdot J \cdot V}{A_{\text {rec }} \sin ^{2}\left(\theta_{\text {sun }}\right) \cdot \sigma \mathrm{T}_{\text {sun }}^{4}}=\frac{A_{\text {cells }}}{A_{\text {emit }}} \cdot \frac{A_{\text {emit }}}{A_{\text {abs }}} \cdot \frac{C_{\text {max }}}{C} \cdot \frac{1}{\sigma T_{\text {sun }}^{4}} \cdot J \cdot V$

where $\sigma$ is the Stefan-Boltzmann constant. In this equation, $J$ has a very complex dependence on the full system configuration $\left(C, \varepsilon_{\mathrm{ca}}, \varepsilon_{\mathrm{gap}}, \varepsilon_{\mathrm{ce}}, A_{\mathrm{emi}} / A_{\mathrm{abs}}\right.$, etc. ) because it depends on the emitter temperature $T_{\text {ernit, }}$ which is obtained from Eq. (14), which includes all these variables.

\section{Methods and results}

The first step must be to solve Eq. (14), which provides the absorber/emitter equilibrium temperature ( $T_{\text {emit }}$ ). To obtain a unique solution for this equation, a set of 10 variables must be fixed. These variables are: the concentration factor ( $C$ ), absorber cut-off energy $\left(\varepsilon_{\mathrm{ca}}\right)$, emitter cut-off energy $\left(\varepsilon_{\mathrm{ce}}\right)$, band-gap energy of the semiconductor $\left(\varepsilon_{\text {gap }}\right)$, PV cell voltage $(V)$, emitter-to-absorber area ratio $\left(A_{\text {emit }}\right)$ $\left.A_{\text {abs }}\right)$, emitter-to-cells area ratio $\left(A_{\text {emit }} / A_{\text {cells }}\right)$, BSR reflectivity $\left(\rho_{\mathrm{bss}}\right)$, emitter-to-cells view factor $\left(F_{\mathrm{ec}}\right)$ and cells-to-cells view factor $\left(F_{\mathrm{oc}}^{\text {(e) }}\right)$. Therefore, the resulting system efficiency (calculated from Eqs. (16) and (17)) depends on all of these variables and the determination of the different dependences becomes too complex. Thus, we first decided to conduct a full-system optimization to find the maximum system efficiency using the simplex (or Nelder-Mead) multidimensional optimization algorithın [20|. This algorithm consists of a direct search to find the optimum combination of a set of variables that locally maximize a merit function (in our case, the system efficiency given by Eq. (17)). Therefore, to avoid local maximums, the algorithm 
must be run for several different sets of initial conditions. In addition, the solution of (14) and (16) requires us to numerically solve the integrals appearing in Eqs. (1) and (2). The numerical solution of these integrals is not trivial because they must be computed over a non-definite interval and their integrands have a singularity when $\varepsilon$ approaches $\mu$. Both problems were overcome by making the variable change $x=k T /(\varepsilon-\mu)$ [21] before composite integration.

To simplify the analysis, we must note that some of the optimum values of the aforementioned variables can be easily predicted. For instance, for an ideal cavity we have:

- $F_{\mathrm{ec}}=1$, which means that the TPV optical cavity is completely closed (without holes) and without any inactive areas; thus, the emitter only sees the cells. Using basic view factor algebra [18|, this condition implies that $F_{c \mathrm{cc}}^{(\mathrm{e})}=1-A_{\text {emit }} / A_{\text {cells }}$.

- $\rho_{\text {bs }}=1$, which means that no photons are lost in the BSR

- $F_{\mathrm{cc}}^{\mathrm{e}}=0$ (or equivalently $A_{\text {emit }} / A_{\text {cells }}=1$, taking into account the first assumption) because it provides the maximum short-circuit photocurrent density (Eq. (16)) and emitter temperature (Eq. (14)).

If these assumptions are taken into account, $\varepsilon_{c e}$ disappears from Eqs. (14) and (16), and therefore, the value of the emitter cut-off energy $\varepsilon_{\mathrm{ce}}$ becomes irrelevant because no photons escape from the cavity; consequently, all the photons with $\varepsilon<\varepsilon_{c e}$ are reabsorbed by the emitter. Therefore, the ideal cavity case can be described using only five variables: $C, V, \varepsilon_{\text {gap }}, \varepsilon_{\text {ca }}$ and $A_{\text {emit }} / A_{\text {abs }}$. Thus, the ideal STPV system represents the case in which all five variables are optimized, as discussed in Section 4.1. In addition, two kinds of non-ideal STPV systems are analyzed in Section 4.2. The special case in which the emitter-to-absorber area ratio equals one (representing a planar STPV system) is analyzed in Section 4.2.1, while assuming an ideal TPV cavity, and finally, the non-ideal cavity case is presented in Section 4.2 .2 and analyzed using $F_{\mathrm{ec}}<1$.

\section{Discussion}

\subsection{The ideal STPV system}

For our purposes, an ideal STPV system represents the situation in which (1) the TPV cavity is ideal (see Section 3) and (II) all the system variables are optimized (i.e., they provide the maximum system efficiency). From (I), all the photons that do not generate electron-hole pairs are reabsorbed at the emitter. Nevertheless, two kinds of losses remain in this ideal situation: the emission losses from the absorber to the sun and the sky (ELA) and the thermalization losses of the generated electrons from the high energy states to the edge of the conduction band when the photons have $\varepsilon>\varepsilon_{\text {gap }}$ (TLE). Therefore, a trade-off between these two kinds of losses exists and must be considered to obtain the maximum efficiency, i.e. condition (II).

It is well known from black-body theory that as $\varepsilon_{\text {gap }} / T_{\text {emit }}$ increases, the fraction of emitted energy by a black-body at a temperature $T_{\text {emit }}$ above $\varepsilon_{\text {gap }}$ diminishes and is close to zero when $\varepsilon_{\text {gap }} / T_{\text {emit }}$ approaches approximately $1 \mathrm{meV} / \mathrm{K}$. Consequently, for high $\varepsilon_{\text {gap }} / T_{\text {emir }}$ the TLE losses become less relevant (due to the narrower spectral range, relative to the spectral range of a blackbody emission, of the radiation exchanged between the cells and the emitter) and the ELA losses limit the efficiency. In contrast, for low $\varepsilon_{\text {gap }} / T_{\text {emit }}$ the TLE losses becomes more relevant.

Figs. 6-8 show the maximum efficiency, the electric power density and the emitter temperature as a function of the band-gap energy, for different concentration factors and when the rest of variables are optimized; that is, $A_{\mathrm{emit}} / A_{\mathrm{abs}}, \varepsilon_{\mathrm{ca}}$ and $V$ (i.e., $V=V_{\mathrm{mpp}}$ ).
The optimum values of these variables are shown in Figs. 9, 10 and 12 respectively. Fig. 13 shows the resulting parameter $\varepsilon_{\text {gap }} / T_{\text {emit }}$ after full optimization.

Firstly, for a given band-gap energy, the efficiency (Fig. 6) increases with concentration, and it reaches its maximum for full concentration (i.e., $C=C_{\max }$ ) where the ELA losses are minimized. In addition, the effect of concentration on the maximum efficiency is much more relevant for high band-gap energies because the ELA losses have a greater impact on the efficiency than the TLE losses (because $\varepsilon_{\text {gap }} / T_{\text {emit }}$ is very high). In contrast, for low band-gap energies, the efficiency becomes almost independent of concentration, although it still has a great effect on the electric power density (Fig. 7).

Secondly, the efficiency (Fig. 6) monotonically increases with the band-gap energy, which is a result of the increase in $\varepsilon_{\text {gap }} / T_{\text {emir }}$ (Fig. 13) that represents a quasi-monochromatic exchange

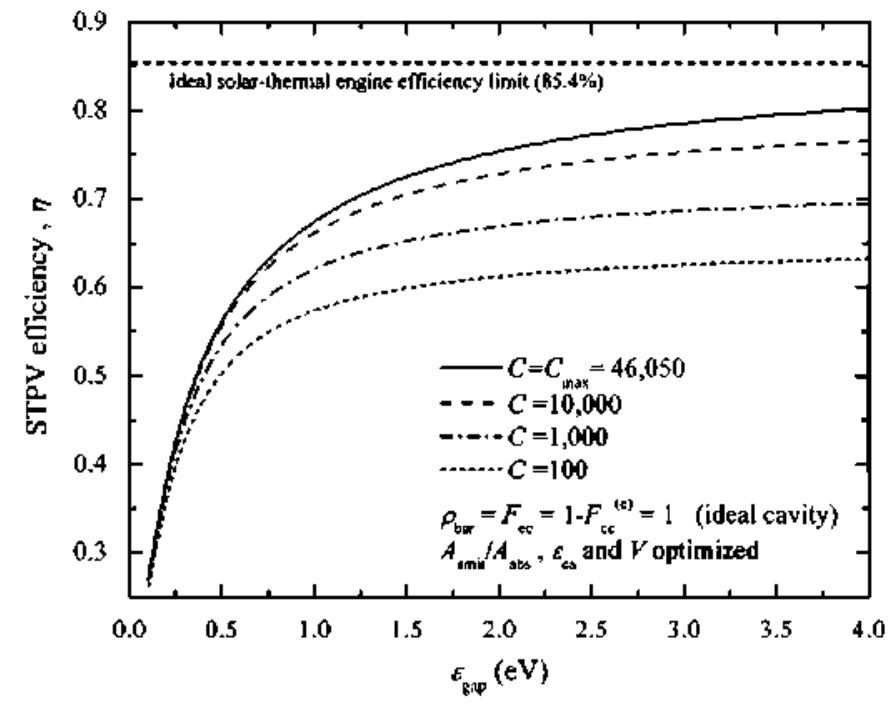

Fig. 6. The maximum STPV elficiency as a function of the band-gap energy ( $\left.\varepsilon_{\text {gap }}\right)$ for different concentration factors (C). The cells voltage ( $V$ ), the emitter-toabsorber area ratio $\left(A_{\text {emit }} / A_{\text {abs }}\right)$ and the absorber cut-olf energy $\left(\varepsilon_{c a}\right)$ have been optimized for each point of these curves.

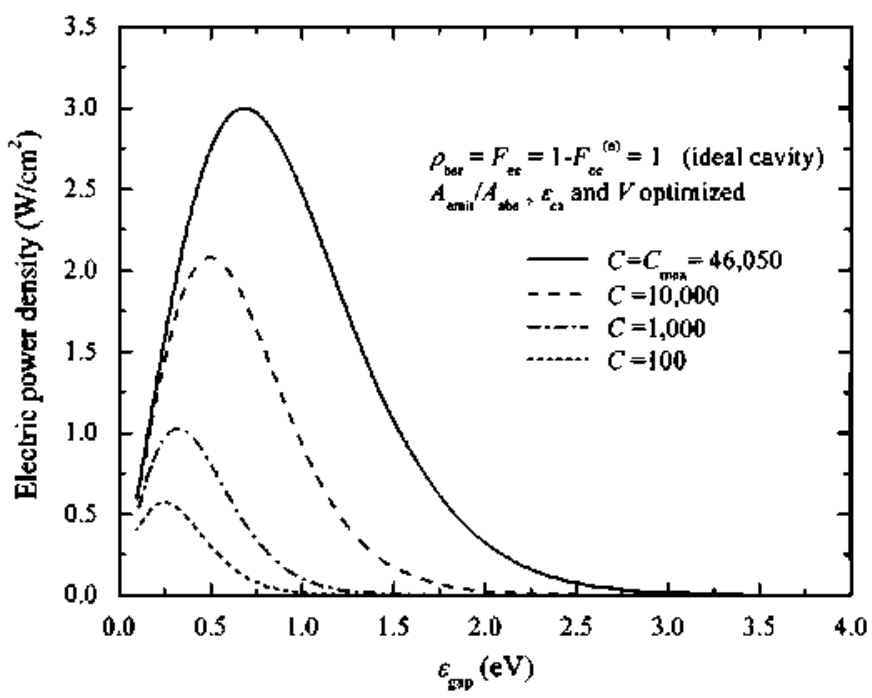

Fig. 7. The electrical power density at the maximum power point of the IV curve $\left(V=V_{\text {mpp }}\right)$ for different concentration factors as a function of band-gap energy $\left(\varepsilon_{\text {gap }}\right)$, obtained under optimized emitter-to-absorber area ratio $\left(A_{\text {emit }} / A_{a b s}\right)$ and absorber cut-of energy $\left(\varepsilon_{\mathrm{ca}}\right)$. 


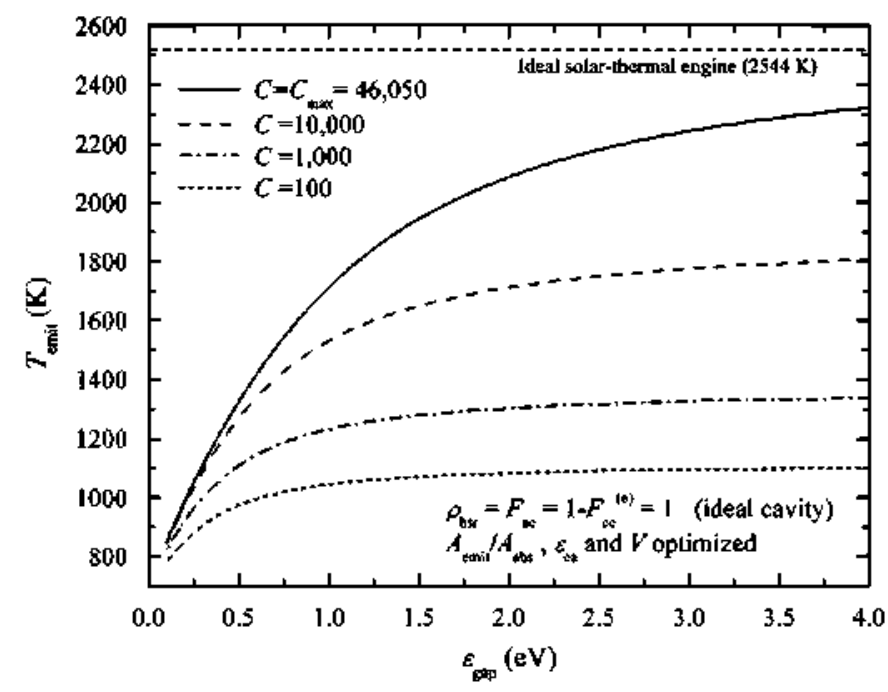

Fig. 8. The emitter temperature ( $T_{\text {emo }}$ ) for diferent concentration factors as a function of band-gap energy ( $\left.\varepsilon_{g a p}\right)$, obtained under optimized cells voltage ( $V$ ). emitter-to-absorber area ratio $\left(A_{\text {emit }} j A_{a b s}\right)$ and absorber cut-off energy $\left(\varepsilon_{c a}\right)$.

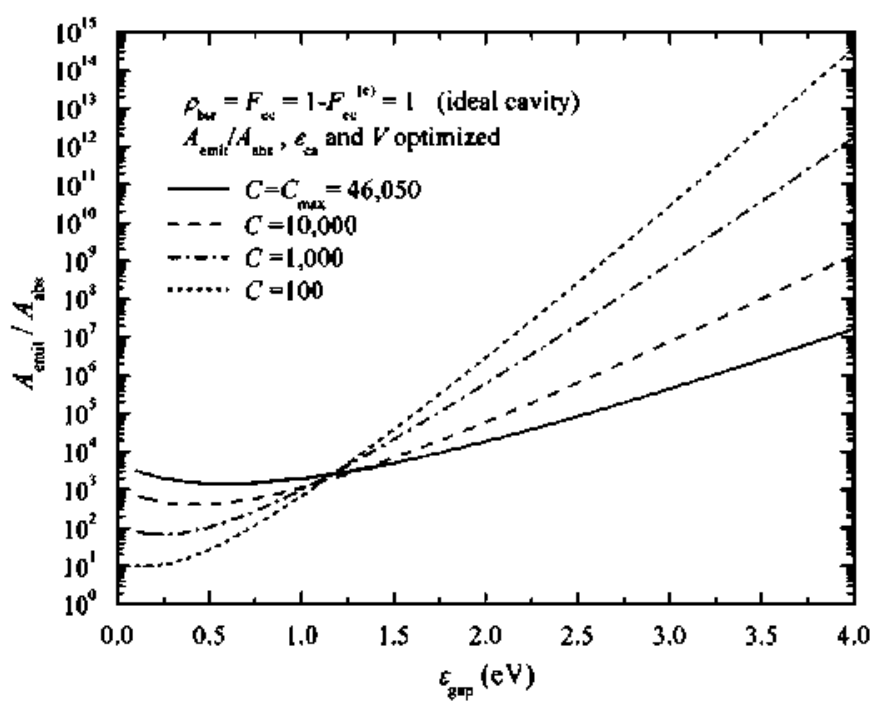

Fig. 9. The optimum enitter-to-absorber area ratio $\left(A_{\mathrm{emu}} / A_{\mathrm{abs}}\right)$ for dilTerent concentration factors as a function of band-gap energy $\left(\varepsilon_{\text {gap }}\right)$.

between the cells and the emitter. Nevertheless, this situation requires a very large emitter-to-absorber area ratio (Fig. 9) to avoid excessive ELA losses. Because of it, the system has a very low electric power density (Fig. 7), which reveals the existence of a trade-off between efficiency and power density (already identified [7]) and represents one of the fundamental barriers to reaching high efficiency in these kinds of STPV systems: the maximum efficiency is obtained when the electric power density approaches zero.

Because the results shown in Figs. 6-8 are obtained for a set of optimized variables (i.e., cell voltage $V$, absorber cut-off energy $\varepsilon_{\mathrm{ca}}$ and the emitter-to-cells area ratio $A_{\text {emit }} / A_{a b s}$ ), it is interesting to analyze the effect of each of these variables separately.

\subsubsection{PV cells voltoge}

In STPV systems, the photons emitted by the cells due to radiative recombination (photons with $\mu=q V$ ) can be absorbed

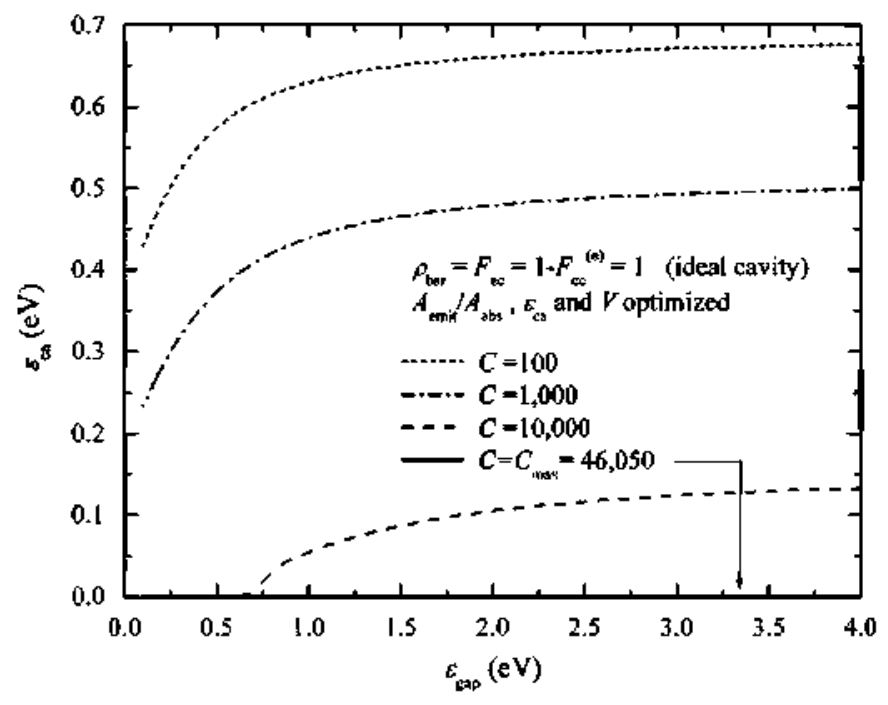

Fig. 10. The optimum absober cut-olf energy $\left(\varepsilon_{c a}\right)$ for different concentration factors as a function of band-gap energy $\left(\varepsilon_{g a p}\right)$.

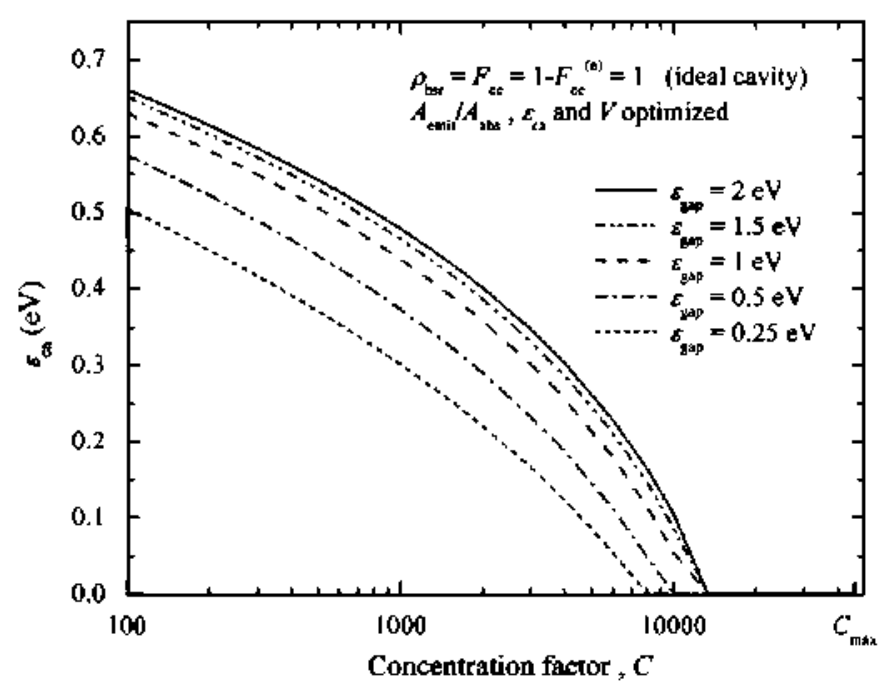

Fig. 11. The optimum absorber cut-olf energy $\left(\varepsilon_{c a}\right)$ for different band-gap energies (Egap) as a function of the concentration factor.

by the emitter. As a result, the equilibrium emitter temperature depends on the cell voltage (this is consistent with the presence of $V$ in Eq. (14)). Therefore, the resulting optimum cell voltage $V_{\text {mpp }}$ is derived from a complex trade-off between generation, recombination and the resulting emitter equilibrium temperature (which is obtained from Eq. (14) and also depends on the full system configuration).

In order to analyze the resulting optimized voltage, it is convenient to formulate $q V_{\text {mpp }} / \varepsilon_{\text {gap }}$ (Fig. 12) as the product of two factors: $\left(q V_{o c} / \varepsilon_{\text {gap }}\right)$ and $\left(V_{\text {mpp }} / V_{o c}\right)$ shown in Figs. 14 and 15 , respectively. First, it must be noted that the low $V_{\text {mpp }}$ values obtained for low concentrations are attributed to a $V_{o c}$ decrease (Fig. 14) that results from a reduction of the carrier generation rate. Second, Fig. 12 shows that $V_{\text {mpp }}$ increases with band-gap energy because $V_{\text {mpp }}$ approaches $V_{o c}$ (Fig. 15), although $V_{o c}$ is slightly reduced with respect to the band-gap energy (Fig. 14). This situation represents an increase in the radiative recombination at the diode active layers, because $\varepsilon-q V_{\text {mpp }}$ (the exponent of the exponential function of Eqs. (1) and (2)) is 


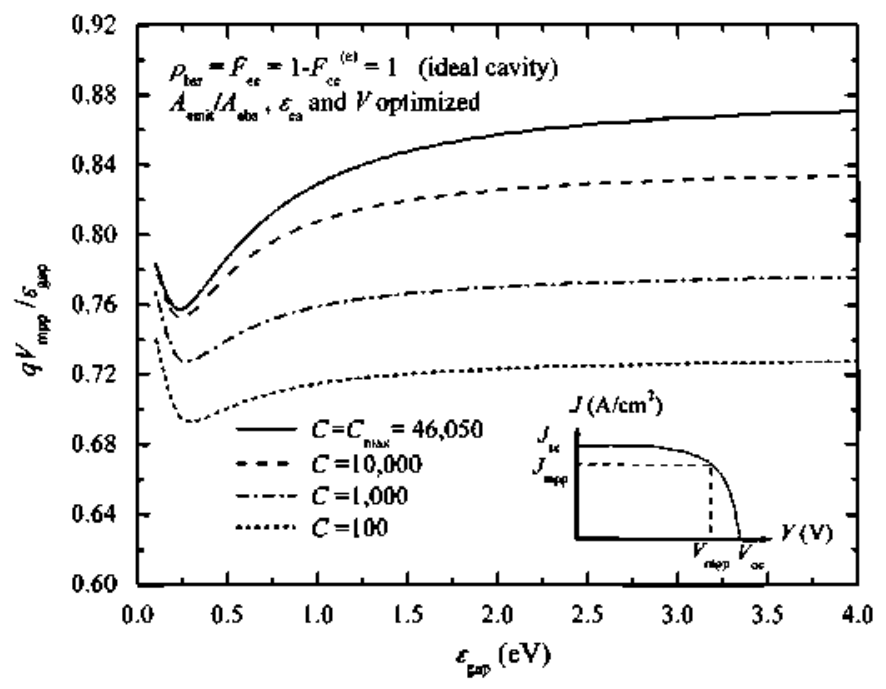

Fig 12. The optimum cells voltage $V_{\text {mpp }}$ for diferent concentration factors as a function of band-gap energy ( $\left.\varepsilon_{\text {gap }}\right)$.

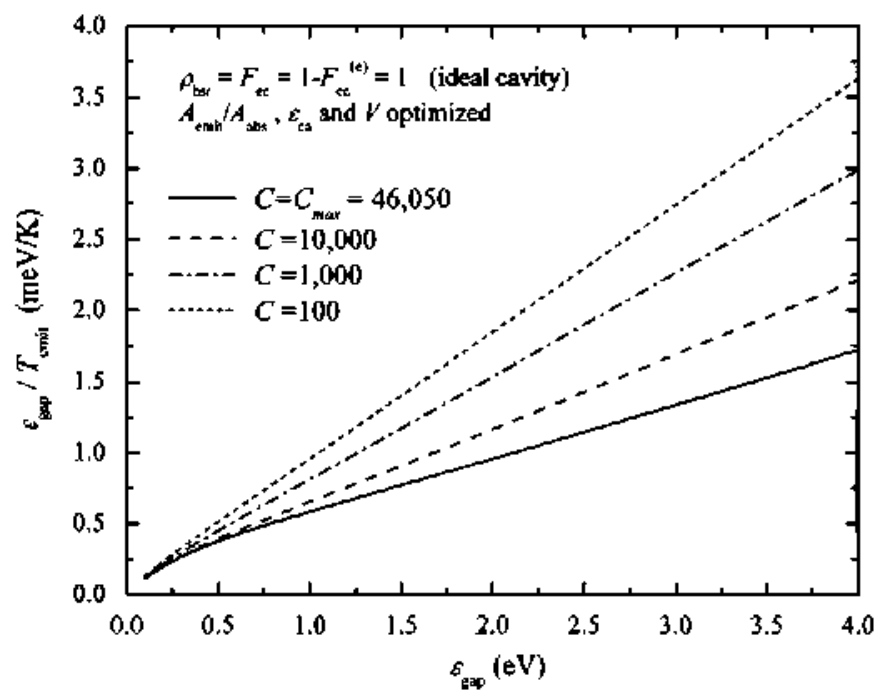

Fig. 13. The optimum $\varepsilon_{\text {gap }} / T_{\text {emlt }}$ for dilferent concentration factors as a function of band-gap energy $\left(\varepsilon_{\mathrm{gpp}}\right)$.

decreased. Consequently, the radiative recombination contribution to heat the emitter is maximal for high band-gap energies. Nevertheless, at very low band-gap energies, $V_{\text {mpp }}$ shows an unexpected increase, which must be attributed to a sharp increase in the open-circuit voltage (Fig. 14) that appears due to the increase in the carriers generation rate at low band-gap energies [7].

\subsubsection{Absorber cut-off energy}

Both the concentration optics $(C)$ and the spectral selective absorber ( $\left.\varepsilon_{\mathfrak{c a}}\right)$ make up the sunlight receptor system (Section 2.2 ), and their function is to minimize the ELA losses. The optimum $\varepsilon_{\text {ca }}$ is shown in Figs. 10 and 11 as a function of the band-gap energy and concentration factor, respectively. When $C=C_{\max }$, the absorber only exchange photons with the sun; thus, the ELA losses cannot be further minimized, and the optimum $\varepsilon_{c a}$ is zero. In other words, a black-body absorber represents the best alternative when the concentration factor is close to $C_{\max }$. However, for $C \& C_{\max }$ the use of a spectral selective absorber is

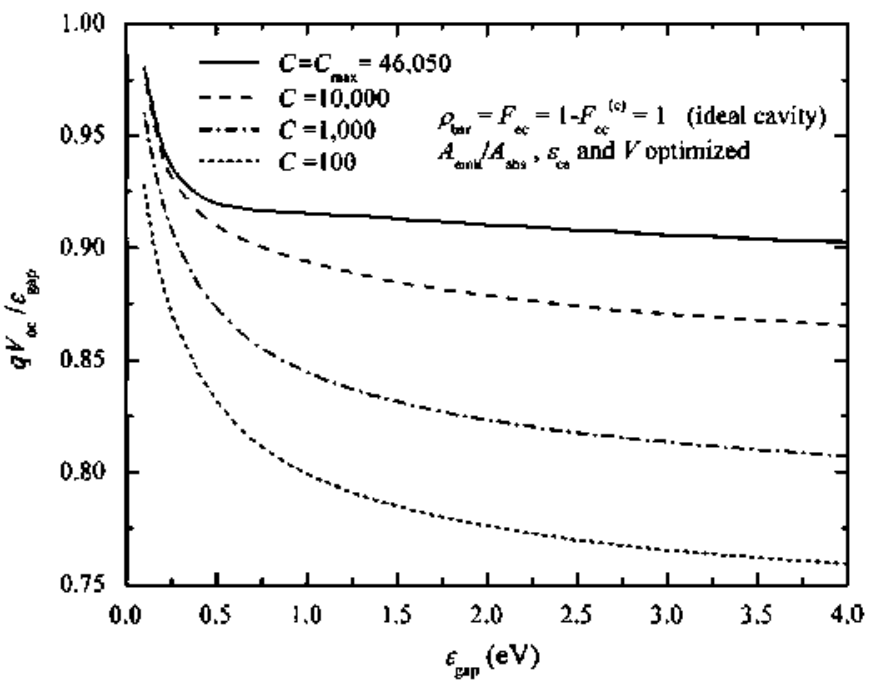

Fig. 14. The open-circuit voltage divided by the band-gap energy as a function of band-gap energy.

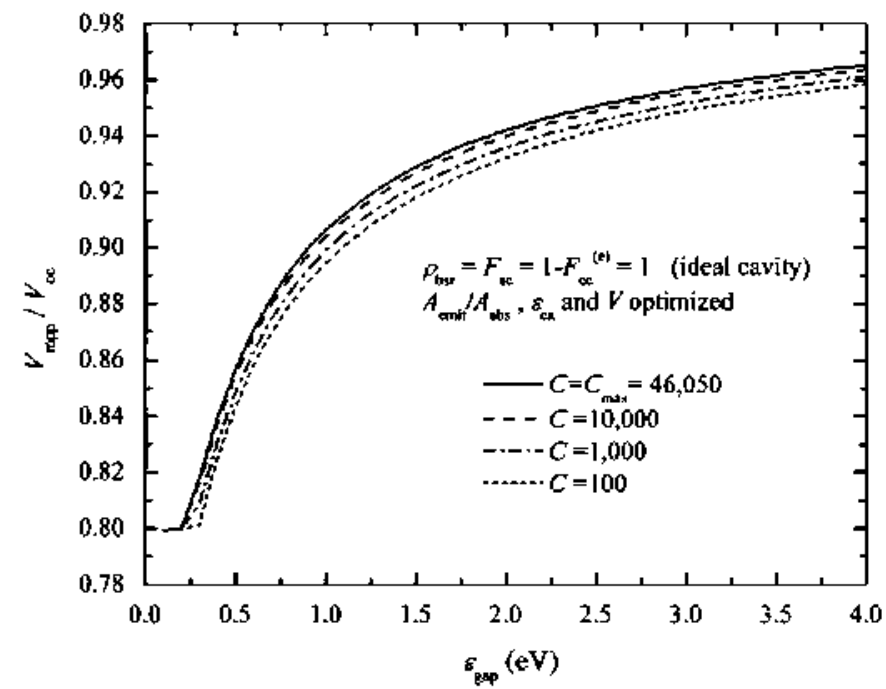

Fig. 15. The maximum power point voltage divided by open-circuit voltage as a function of band-gap energy.

required because in this situation, the absorber also exchanges photons with the sky (which is much colder than the sun). and consequently a selective absorber is required to avoid excessive ELA losses. Therefore, spectrally selective absorbers operates better at low concentrations, as was demonstrated previously in [15].

It is also noted that the optimum $\varepsilon_{\mathrm{ca}}$ diminishes as the bandgap energy decreases to obtain a lower $T_{\text {emit }}$ (Fig. 8) and to avoid a dramatic decrease of the parameter $\varepsilon_{\text {gap }} / T_{\text {emit }}$ (Fig. 13), which minimizes the TLE losses that become dominant at lower bandgap energies.

\subsubsection{Emitter-to-obsorber oreo racio}

As we have previously noted, the STPV efficiency monotonically increases with the band-gap energy because the TLE losses are minimized. In this situation, the cells work almost like a perfect reflector, absorbing only a small portion of the photon flux $\left(\varepsilon>\varepsilon_{\mathrm{gap}}\right)$. If in this situation the emitter area were kept low $\left(A_{\text {emit }} \sim A_{\text {abs }}\right.$ ), almost all the power that the emitter/absorber 
component must radiate would be performed by the absorber side instead of the emitter one, increasing the ELA losses. Therefore, to compensate for this effect, $A_{\text {emit }} / A_{\text {abs }}$ must be increased (Fig. 9) in order to maintain an optimum balance between the net rate of photon absorption by the cells and the photon emission by the absorber. This leads to the very high $A_{\text {emit }} / A_{\text {abs }}$ and very low electrical power density obtained at high band-gap energies shown in Figs. 9 and 7 , respectively. Consequently, as $\varepsilon_{\text {gap }}$ goes to lower values both the maximum efficiency and the optimum $A_{\text {emit }} / A_{\mathrm{abs}}$ are reduced, and therefore the power density increases.

Nevertheless, when $\varepsilon_{\text {gap }}$ falls below a certain value (which depends on the concentration factor), the optimum $A_{\text {emit }} / A_{\text {abs }}$ stops decreasing and it statts to slightly increase (Fig. 9). As a result, $T_{\text {ernit }}$ (Fig 8) decreases, which prevents a dramatic decrease of the parameter $\varepsilon_{\text {gap }} / T_{\text {emir }}$ (Fig. 13), which could represent excessive TLE losses. This effect is stronger for high concentration factors because they provide higher emitter temperatures. Consequently, the bandgap energy at which the optimum $A_{\text {emit }} / A_{a b s}$ is smallest, decreases with concentration. Therefore, different behavior occurs when $\varepsilon_{\text {gap }} \leqslant 1.4 \mathrm{eV}$ (where the lowest optimum $A_{\text {emit }} / A_{\text {abs }}$ is obtained for low concentration factors) and when $\varepsilon_{\text {gap }} \geq 1.4 \mathrm{eV}$ (where the lowest optimum $A_{\text {ernit }} / A_{\text {abs }}$ is obtained for high concentration factors).

\subsection{Non-ideal STPV systems}

\subsubsection{Planar configuration}

As discussed in Section 4.1.3, the optimum $A_{\text {emit }} / A_{\text {abs }}$ is usually very high (especially for large $\varepsilon_{\text {gap }}$ ) and therefore, the planar STPV system $\left(A_{\text {emit }} / A_{\text {abs }}=1\right)$ does not represent the optimum design. However, from a practical point of view a large emitter-toabsorber area ratio is not desirable because it causes problems concerning the TPV optica] cavity design [10]. From this perspective, the planar STPV system appears to be a desirable design and it must be analyzed.

Fig. 16 shows how the maximum efficiency varies as $A_{\text {emit }} / A_{\mathrm{abs}}$ decreases from its optimum value down to one (the planar STPV system) for three different band-gap energies. In the case of $\varepsilon_{\text {gap }}=3.5 \mathrm{eV}$, the optimum $A_{\text {ernit }} / A_{\text {abs }}$ is vely high; thus, its reduction to one represents a dramatic difference from the maximum achievable system efficiency due to a significant

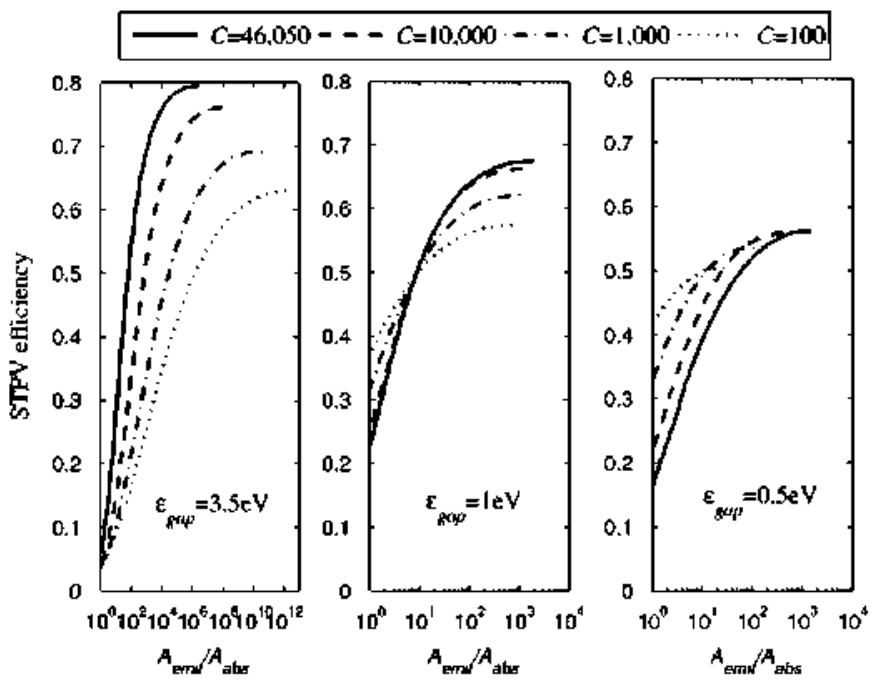

Fig. 16. The maximum STPV system elficiency as a function of $A_{\text {emit }} / A_{\text {abs }}$ for different concentration factors and for three band-gap energies: $3.5 \mathrm{eV}$ (left) $1 \mathrm{eV}$ (center) and $0.5 \mathrm{eV}$ (light). The absorber cut-ol energy $\left(\varepsilon_{\mathrm{ca}}\right)$ is optimized for each point of these curves. increase in the ELA losses. Nevertheless, the maximum efficiency is always obtained for $C=C_{\max }$ as long as the ELA losses still limit the efficiency for all $A_{\text {emit }} / A_{\text {abs. }}$ In contrast, in the case of $\varepsilon_{\text {gap }}=0.5 \mathrm{eV}$ (where the TLE losses dominate), the results shown that for low $A_{\text {emit }} / A_{\text {abs, }}$ the maximum efficiency is obtained under lower concentration factors because under low concentrations, $T_{\text {emit }}$ is lower, $\varepsilon_{\text {gap }} / T_{\text {emit }}$ increases, and therefore, TLE losses are reduced.

Fig. 17 shows the maximum efficiency for the limit situation in which $A_{\text {emit }} / A_{\text {abs }}=1$ (a planar STPV system) as a function of bandgap energy and for different concentration factors, where $V$, and $\varepsilon_{c a}$ are optimized. The corresponding electrical power density is shown in Fig. 18. Both figures show the optimized concentration factor case, which maximizes the system efficiency for a given band-gap energy. In this case, a sharp change in the electrical power density occurs around $\varepsilon_{\text {gap }} \sim 2 \mathrm{eV}$, due to the change of the optimum concentration factor from one (for $\varepsilon_{\text {gap }} \leqslant 2 \mathrm{eV}$ ) to $C_{\max }$

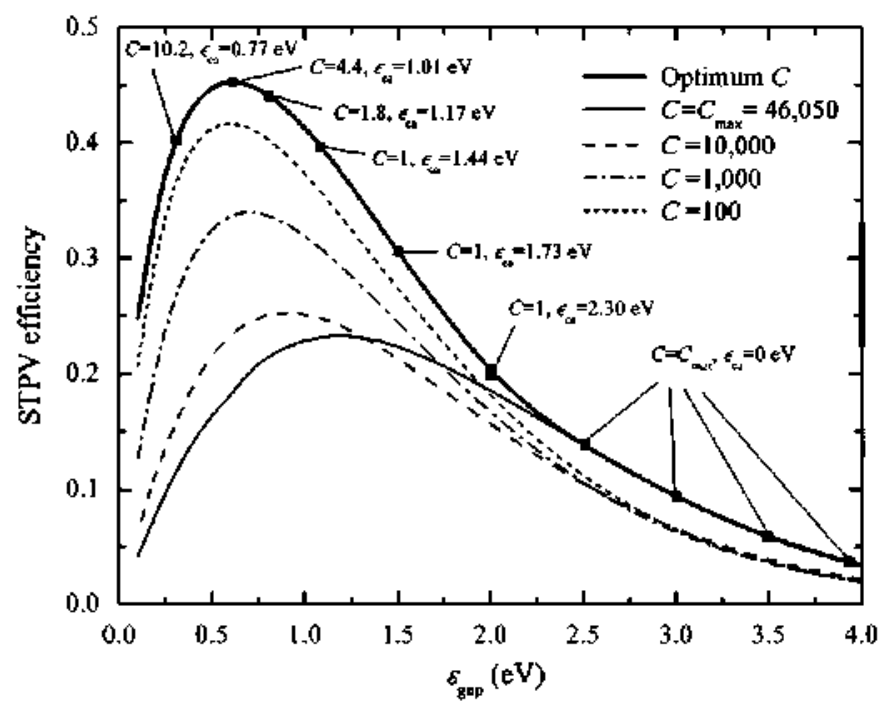

Fig 17. The maximum STPV efficiency for a planar STPV system $\left(A_{\text {emit }} / A_{\text {abs }}=1\right)$ for diflerent concentration factors. The absorber cut-of energy $\left(\varepsilon_{c a}\right)$ is optimized for each point of these curves.

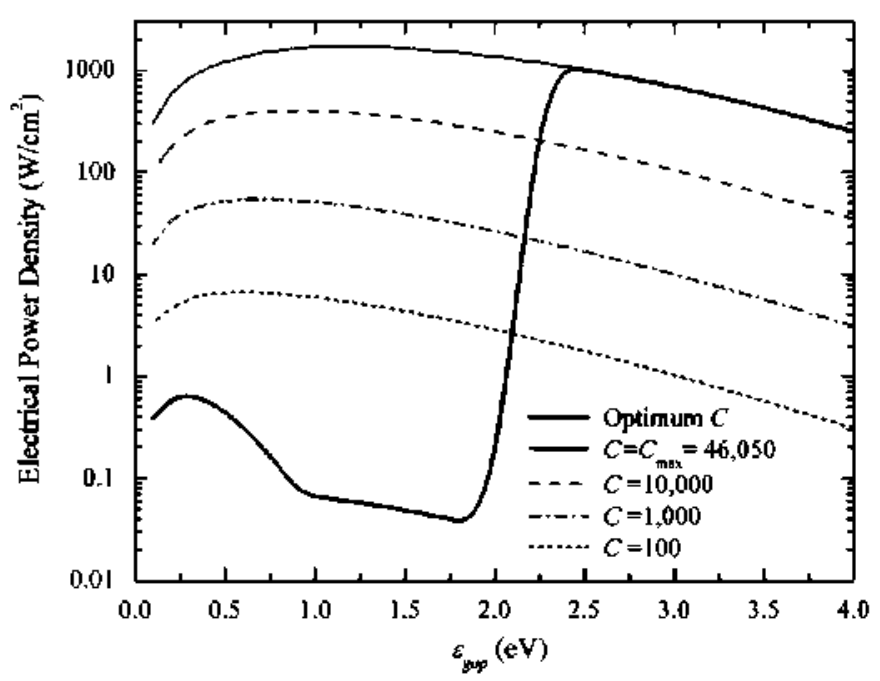

Fig. 18. The electrical power density for a planar STPV system $\left(A_{\text {emit }} / A_{a b s}=1\right)$ for diferent concentration factors. The absorber cut-ol energy $\left(\epsilon_{a}\right)$ is optimized for each point of these curves. 
(for $\varepsilon_{\text {gap }} \geq 2 \mathrm{eV}$ ). This sharp change occurs because a local maximum for the system efficiency exists when $C=C_{\max }$ that becomes global when $\varepsilon_{\text {gap }} \geq 2 \mathrm{eV}$. This behavior illustrates the trade off between ELA and TLE losses; when the band-gap energy is high enough, the TLE losses are negligible, and therefore, the optimum system configuration requires minimizing the ELA losses by using the maximum concentration factor.

From Fig. 17, we can conclude that, as expected after the previous explanations, the maximum efficiency (45.3\%) is obtained under low concentration factors $(C=4.4)$, low bandgap energies ( $\varepsilon_{\text {gap }}=0.6 \mathrm{eV}$ ) and using spectral selective absorbers (with $\varepsilon_{c a}=1.01 \mathrm{eV}$ ). It is remarkable that this efficiency exceeds the Shockley-Queisser limit of 40.8\% [13] (calculated at maximum concentration, optimum band-gap and approximating the sun spectrum to be that of a black body at $6000 \mathrm{~K}$ ), which represents the maximum efficiency for direct conversion of sun radiation using single band-gap PV cells. In addition, the maximum efficiency is obtained at a very low concentration factor (4.4 suns), in contrast to the maximum concentration required for the Shockley-Queisser limit.

This improvement is attributed to the differences in the nature of the light that is returned to the sun by a directly sunilluminated PV cell and by a cell illuminated by an intermediate thermal absorber/emitter. In the first case, which leads to the Shockley-Queisser limit, the emission from the cell to the sun consists of luminescent radiation (with $\mu=q V$ ), which usually represents very high radiative energy fluxes for energies above the band-gap of the cell $\varepsilon_{\text {gap }}$. In contrast, in the second case, the emission from the absorber consists of thermal radiation (with $T=T_{\text {emit }}$ ) that can be minimized using the proper combination of the absorber cut-off and concentration factor.

Fig. 18 shows that the maximum efficiency is obtained for a relatively low electrical power density (compared with the others combinations of concentration and absorber cut-off) of $\sim 0.32 \mathrm{~W} / \mathrm{cm}^{2}$. Therefore, the trade-off between efficiency and power density explained in the previous section still remains in the planar configuration. Nevertheless, the combination of Figs. 17 and 18 show that both high efficiency (30-40\%) and high electrical power density $\left(1-100 \mathrm{~W} / \mathrm{cm}^{2}\right)$ can be obtained under concentration ratios between 100 and 1000 if the spectrally selective absorber is properly designed.

\subsubsection{Non-ideal TPV cavity}

Two kinds of losses are attributed to a non-ideal TPV cavity: (I) absorption at the back-side reflector $\left(\rho_{\mathrm{bsr}}<1\right)$ and (II) photon loss outside the cavity $\left(F_{\mathrm{ec}}<1\right)$. In the second case, both $F_{\mathrm{ec}}$ and $F_{c c}^{(e)}$ are functions of the dimensions of the specific cavity geometry, making it difficult to provide general conclusions. Only assuming that the cells cannot see each other (i.e., $F_{c c}^{(e)}=0$ ), for instance, when cells are placed very close to the emitter, allows this case to provide general results as a function of $F_{e c}$ only. Futhermore, it can be deduced from Eq. (14) that in the case of using an ideal cut-off emitter with $\varepsilon_{\text {ce }}=\varepsilon_{\text {gap }}, \rho_{\text {bss }}$ does not affect the system performance because the last term of Eq. (14) vanishes. In contrast, if a black-body emitter $\left(\varepsilon_{\mathrm{ce}}=0\right)$ is used (and assuming $F_{c c}^{(e)}=0$ ), $F_{e c}$ has a greater impact in the system performance than $\rho_{\text {bsr }}$ because it appears squared in Eq. (14). For all of these reasons, we will only evaluate the impact of $F_{\mathrm{ec}}$ on the system efficiency for two cases: a black-body emitter $\left(\varepsilon_{\text {ce }}=0\right)$ (Fig. 19) and a perfect cut-off emitter with $\varepsilon_{\mathrm{ce}}=\varepsilon_{\mathrm{gap}}$ (Fig. 20). In both cases, $C=C_{\max }$ is assumed to minimize the ELA losses, which are out of the scope of this section.

In both cases, the cavity losses have a greater impact on the system efficiency when the band-gap is high; this impact is much more relevant in the black-body emitter case (Fig. 19). For

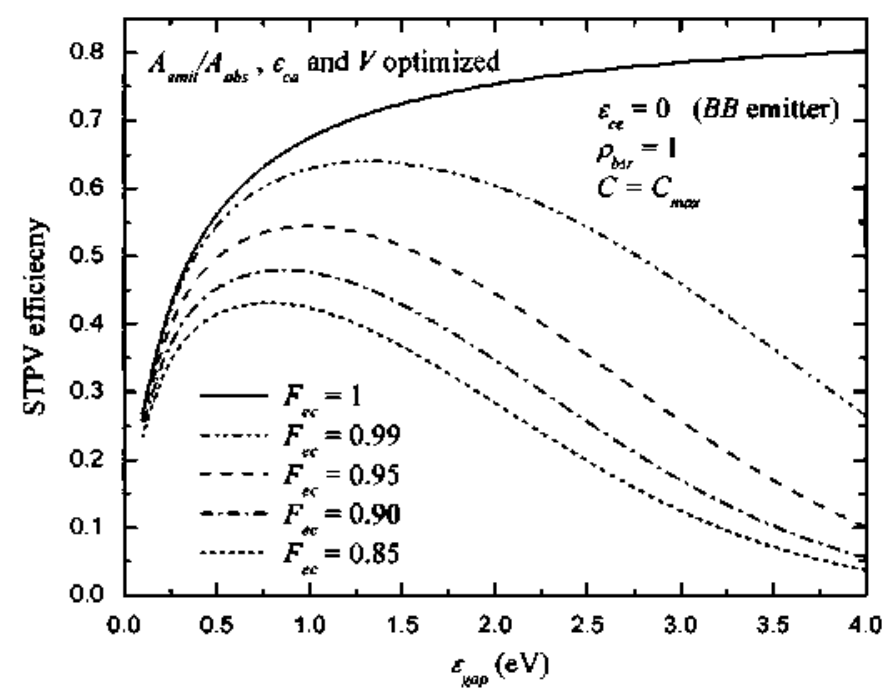

Fig. 19. The maximum STPV elficiency for dilferent emitter-to-cells view factors $\left(F_{e c}\right)$ when the cells voltage $(V)$, the emitter-to-absorber area ratio $\left(A_{\text {emit }} / A_{\text {abs }}\right)$ and the absorber cut-off energy $\left(\varepsilon_{\mathrm{ca}}\right)$ are optimized for each point of these curves, and $c=C_{\max }, \rho_{\text {brr }}=1$ and $\varepsilon_{\mathrm{ce}}=0$ (representing a black-body emitter). The case $f_{\mathrm{ec}}=1$ represents the ideal cavicy case also shown in Fig. $\sigma$ for $C=C_{\max }$.

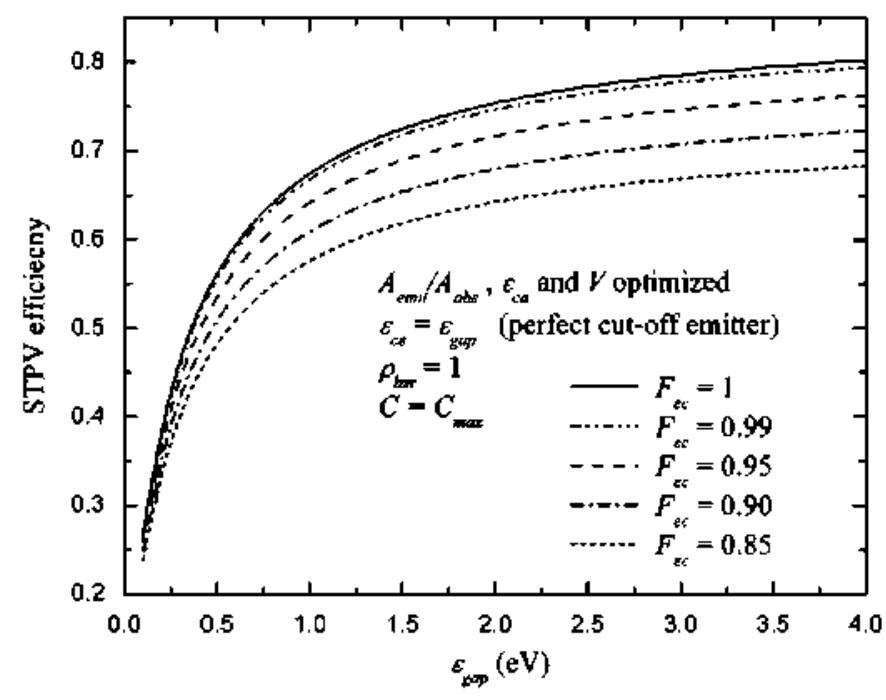

Fig. 20. Maximum STPV eॉriciency for dillerent emitter-to-cells view factors ( $\left.F_{\text {ec }}\right)$ when the cells voltage $(V)$, the emitter-to-absorber area ratio $\left(A_{\text {emut }} / A_{\text {abs }}\right)$ and the absober cut-off energy $\left(\varepsilon_{c a}\right)$ are optimized for each point of these curves, and $C=C_{\max }, \rho_{\text {bsr }}=1$ and $\varepsilon_{c e}=\varepsilon_{\text {gap }}$ (representing a perfect cut-off emitter). The case $F_{\mathrm{ec}}=1$ represents the ideal cavicy case also shown in Fig. 6 for $C=C_{\max }$.

instance, in the black-body emitter case (Fig. 19), if we consider the case of $\varepsilon_{\text {gap }}=4 \mathrm{eV}$ and a view factor of $99 \%\left(F_{\mathrm{ec}}=0.99\right)$ between the emitter and the cells (which means that only $1 \%$ of the areas in the cavity are inactive), the limiting efficiency drops from approximately $80 \%$ (ideal cavity case) to approximately $30 \%$. However, when $\varepsilon_{\text {gap }}=1 \mathrm{eV}$, the efficiency only drops from approximately $67 \%$ to approximately $63 \%$. Furthermore, the optimum band-gap decreases as the cavity losses increase.

As expected, the impact of $F_{e c}$ on the system efficiency is reduced when using a perfect cut-off emitter (Fig. 20) because the radiation lost outside the cavity is restricted to photons with $\varepsilon>\varepsilon_{c e}=\varepsilon_{\text {gap }}$. Nevertheless, realistic emitters do not behave as perfect cut-off emitters, and they show a non-zero emissivity for $\varepsilon>\varepsilon_{\text {cee }}$, which means that in realistic cases, the efficiency drop 
due to cavity losses varies from the best case of having a perfect cut-off emitter (Fig. 20) down to the worst case of having a black-body emitter (Fig. 19).

\section{Summary and conclusions}

In this paper we have presented a detailed balance analysis of an STPV system that consists of a sunlight receptor system (optical concentrator and a cut-off broad band absorber), a cut-off broad band emitter and single junction solar cells working in the radiative limit with an integrated back-side reflector, in a configuration where the cells enclose the emitter forming a TPV optical cavity. In this analysis, 10 variables have been considered: concentration factor, absorber and emitter cut-off energies, the band-gap energy of the PV cells, the PV cell voltage, the emitterto-absorber and emitter-to-cells area ratios, the reflectivity of the back-side reflector and, finally, the emitter-to-cells and cells-tocells view factors. All these variables affect the resulting emitter temperature (extracted from the solution of the energy balance equation at the absorber/emitter component); thus, analysis of this kind of system requires a complex multi-variable treatment, which has been proposed in this paper.

We found that the limit efficiency monotonically increases with the band-gap energy when the rest of the variables are optimized and there are no optical losses within the TPV cavity. As the band-gap energy increases, the efficiency limit approximates (but is always lower than) that of an ideal solar therinal engine (85.4\%). Nevertheless, this situation requires a very high emitterto-absorber area ratio $\left(\geq 10^{7}\right)$, and consequently provides very low electrical power density. In addition, in this case, the efficiency drops sharply when a small fraction of photons are lost outside the TPV cavity. In contrast, the use of low band-gap PV cells require a much lower emitter-to-absorber area ratio ( $\lesssim 10^{3}$ ), higher power densities (up to $\sim 3 \mathrm{~W} / \mathrm{cm}^{2}$ ) and the impact of the cavity losses on the system efficiency is drastically reduced. In addition, the efficiency limit in this case can be still very high (up to $\sim 70 \%$ ). For all of these reasons low-band gap PV cells represent a much more convenient alternative for the implementation of real high-efficiency STPV systems, despite their lower efficiency limit.

In addition, we found that the optimum absorber cut-off energy approaches zero when the concentration factor approaches the maximum, which means that a spectrally selective absorber works better for lower concentration factors. Nevertheless, the system efficiency and, importantly, the electrical power density, increase with the concentration factor when the rest of the variables are optimized. As a result, both the maximum efficiency and the maximum electrical power densities are obtained for a combination of maximum concentrations and a black-body absorber.

However, when the emitter-to absorber area ratio is reduced from the optimum, the optimum concentration is no longer the maximum, and the maximum efficiency is obtained for a specific combination of concentration factor (lower than the maximum) and absorber cut-off energy (higher than zero). This phenomenon occurs for an emitter-to-absorber area ratio of one, that is, a planar-STPV system, which represents a very interesting design from a practical point of view. The efficiency limit of planar STPV systems using single junction PV cells is $45.3 \%$, which exceeds the Shockley-Queisser limit of 40.8\% (obtained for a single junction solar cell, directly illuminated by the sun, working under a maximum concentration factor and with an optimized band-gap).
This efficiency is obtained for a very low concentration factor of 4.4 suns and a selective absorber with a cut-off energy of $1.01 \mathrm{eV}$. In addition, it provides a relatively high electrical power density of $0.32 \mathrm{~W} / \mathrm{cm}^{2}$ (compared with the sunlight energy flux at the Earth of $\sim 0.16 \mathrm{~W} / \mathrm{cm}^{2}$ ). In addition, if higher concentration factors were used (from 100 to 1000 suns), the electrical power density of these systems could be drastically increased (from 1 to $100 \mathrm{~W} / \mathrm{cm}^{2}$ ) while maintaining a relatively high system efficiency limit (from $30 \%$ to $40 \%$ ).

\section{Acknowledgments}

The authors gratefully acknowledge E. Antolin and A. Martí for fruitful discussions concerning the fundamental aspects of the detailed balance theory and $G$. del Coso and P. Espinet for their support configuring the work stations on which the simulations were run. A. Datas acknowledges financial support from the 'Consejería de Educación de la Comunidad de Madrid y del Fondo Social Europeo (FSE)'.

\section{References}

[1] A. Luque, S. Hegedus, Handbook of Photovoltaic Science and Engineering, Wiley, 2003.

[2] P. Davis, A. Luque, Solar thermophotovoltaics: brief review and new look, Solar Energy Materials and Solar Cells 33 (1994) 11-22.

[3| A. DeVos, H. Pauwels, On the themodynamic limit of photo-voltaic energyconversion, Applied Physics 25 (2) (1981) 119-125.

|4| R. Winston, J. Minano, P. Benitez, Nonimaging Optics, Elseviel Academic PTess, 2005.

[5] M. Florescu, H. Lee, l. Puscasu, M. Ptalle, L. Florescu, D. Ting, J. Dowling, Improving solar cell efficiency using photonic band-gap materials, Solar Energy Materials and Solar Cells 91 (2007) 1599-1610.

15| A. Luque, J Minano P. Davies, M. Terrón, I Tobias, G Sala, J Alsonso. J Oliván. Angle-limited cavities for silicon solar cells, in: Record of the Twenty Second IEEE Photovoltaic Specialist Conference, IEEE, vols. 1 and 2, 1991, pp. 99-104.

|7| P. Baldasaro, J. Raynolds, G. Charache, D. DePoy, C. Ballinger, T. Donovan, J. Borrego, Thermodynamic analysis of thermophotovoltaic efficiency and power density tradeoIs, Journal of Applied Physics 89 (2001) 3319-3327.

|8| A. Luque, G. Sala, ]. Luque-Heredia, Photovoltaic concentration at the onset of its commercial deployment, Progress in Photovoltaics: Research and Applications 14 (2006) 413-428.

[9] A. Gombert, An overview of tpv emitter technologies, in: Thermophotovoltaic Generation of Electricity, 2003, Proceedings Paper.

|1이 N.-P. Harder, P. Würfel, Theoretical limits of thermophotovoltaic solar energy conversion, Semiconductor Science and Technology 18 (2003) S151-5157.

111 A. Datas, C. Algora, Analytical model of solar thermophotovoltaic systems with cylindrical symmetry: ray tracing approach, Progress in Photovoltaics 17 (2009) 526-541.

|12| W. Shockley. H. Queisser, Detailed balance linit of efficiency of p-n junction solar cells, Joumal of Applied Physics 32 (1961) 510-519.

|13| G. Alaújo. A. Martí, Absolute limiting efficiencies for photovoltaic energy conversion, Solar Energy Materials and Solar Cells 33 (1994) 213-240.

[14] P. Würel, The chemical potential of radiation, Joumal of Physics C-Solid State Physics 15 (1982) 3967-3985.

[15] P. Würfel, W. Ruppel, Upper limit of thermophotovoltaic solar-energy conversion, JEEE Transactions on Electron Devices 27 (1980) 745-750.

[16] N. Sergeant, O. Pincon, M. Agrawal, P. Peumans, Design of wide-angle solarselective absorbers using aperiodic metal-dielectric stacks, Optics Express 17 (2009) 22800-22812.

[17] I. Celanovic, N. Jovanovic, J. Kassakian, Two-dimensional tungsten photonic crystals as selective thermal emitters, Applied Physics letters 92 (2008) 193101 .

[18] R. Siegel, J. Howell, Thermal Radiation Heat Transfer, Mc Graw Hill, 1972.

|19| D.L Chubb. Fundamentals of Thermophotovoltaic Energy Conversion, Elsevier, 2007.

[20] J. Lagarias, J. Reeds, M. Wright, P. Wright, Convergence properties of the Nelder-Mead simplex method in low dimensions, SlAM Journal on Optimization 9 (1) (1998) 112-147.

|21| M. levy. C. Honsberg. Rapid and precise calculations of energy and particle flux for detailed-balance photovoltaic applications, Solid-State Electronics 50 (2006) 1400-1405 